\title{
A simple CMOS chaotic integrated circuit
}

\author{
José L. Rossellóa), Vincent Canals, Ivan de Paúl, Sebastià Bota, \\ and Antoni Morro
}

Electronic Systems Group, Balearic Islands University,

Cra. Valldemossa km. 7.5, Palma de Mallorca 07122, Balearic Islands, Spain

a)j.rossello@uib.es

Abstract: A chaotic integrated circuit is designed and fabricated using a $0.35 \mu \mathrm{m}$ CMOS process. The circuit iterates an N-shaped transfer function using a small analog neural network. One of the advantages of the proposed circuit is its small circuit area with only 13 MOS transistors. The circuit generates both an analog and a digital signal that can be used to create true random bit sequences.

Keywords: Chaotic Circuits, Neural Networks, CMOS

Classification: Integrated circuits

\section{References}

[1] J. Wu, J. Hou, Y. Zhang, and T. Wang, "Secure communications via synchronized chaotic circuit," Proc. Int. Conf. Signal Processing, Beijing, China, vol. 2, pp. 1552-1555, 2002.

[2] A. J. K. Klomkarn and P. Sooraksa, "Further investigation on trajectory of chaotic guiding signals for robotic systems," Proc. Int. Symp. Communications and Information Technologies, Sapporo, Japan, vol. 2, pp. 1166-1170, 2004.

[3] M. Delgado-Restituto and A. Rodriguez-Vazquez, "Integrated chaos generators," Proceedings of the IEEE, vol. 90, pp. 747-767, May 2002.

[4] C. C. Wang, J. M. Huang, H. C. Cheng, and R. Hu, "Switched-current 3bit CMOS 4.0-MHz wideband random signal generator," IEEE J. SolidState Circuits, vol. 40, pp. 1360-1365, June 2005.

[5] M. J. Bellido, A. J. Acosta, M. Valencia, A. Barriga, and J. L. Huertas, "Simple binary random number generator," Electron. Lett., vol. 28, no. 7, pp. 617-618, March 1992.

[6] T. Tanaka and E. Hiura, "Dynamic behavior of a chaotic neural network and its application to optimization problems," Proc. Int. Joint Conf. Neural Networks, Montreal, Canada, pp. 753-757, 2005.

[7] L. O. Chua, "The Genesis of Chua's circuit," Int. J. Electron. Commun., vol. 46, pp. 250-257, 1992.

[8] T. Matsumoto, "A Chaotic Attractor from Chua's Circuit," IEEE Trans. Circuits Syst., vol. CAS-31, no. 12, pp. 1055-1058, Dec. 1984.

[9] J. M. Cruz and L. O. Chua, "An IC Chip of Chua's Circuit," IEEE Trans. Circuits Syst. II, vol. 40, no. 10, pp. 614-625, Oct. 1993.

[10] Y. Hosokawa et. al., "A Design Method of Chaotic Circuits using an Oscillator and a Resonator," Proc. International Symposium Circuits and Systems, vol. III, pp. 373-376, 2001. 
[11] H. Nakano et. al., "A Simple Nonautonomous Chaotic Circuit with a Periodic Pulse-Train Input," Proc. International Symposium Circuits and Systems, vol. III, pp. 108-111, 2003.

[12] A. S. Elwakil, "Nonautonomous pulse-driven chaotic oscillator based on Chua's circuit," Proc. International Symposium Circuits and Systems, vol. III, pp. 136-139, 2003.

[13] A. G. Radwan, A. M. Soliman, and A. L. El-Seedek, "MOS realization of the double-scroll-like chaotic equation," IEEE Trans. Circuits Syst. I, vol. 50, no. 2, Feb. 2003.

[14] E. Lindberg, K. Murali, and A. Tamasevicius, "The smallest transistorbased nonautonomous chaotic circuit," IEEE Trans. Circuits Syst. II, vol. 52, no. 10, pp. 661-664, Oct. 2005.

[15] C. C. Hsu, D. Gobovic, M. E. Zaghloul, and H. H. Szu, "Chaotic neurons models and their VLSI circuit implementations," IEEE Trans. Neural Netw., vol. 7, no. 6, pp. 1339-1350, Nov. 1996.

[16] B. R. Gaines, "Random pulse machines," IEEE Trans. Comput., pp. 410410, April 1968.

[17] J. L. Rosselló, V. Canals, I. de Paúl, and J. Segura, "Using stochastic logic for efficient pattern recognition analysis," Proc. Int. Joint Conf. Neural Networks, pp. 1057-1061, 2008.

[18] M. T. Rosenstein, J. J. Collins, and C. J. De Luca, "A practical method for calculating largest Lyapunov exponents from small data sets," Physica D., vol. 65, pp. 117-134, 1993.

\section{Introduction}

Chaos refers to the impossibility of making accurate long-term predictions about the behavior of non-linear systems. During last decades there has been a high interest in the design and analysis of chaotic systems given their parallelism with nature behavior. In particular, the design and implementation of chaotic circuits has found different fields of application that include secure communications [1], robot control [2], or noise sources implementation [3] frequently employed in speech processing applications or to test the dynamic behavior of electronic systems. Chaotic circuits are also used as random number generators for applications in the security domain of networks and wireless communications [4] and for both analog and digital testing [5]. In particular, neural networks exhibiting a chaotic behavior has been applied to optimization problems [6].

Many chaotic oscillator designs were introduced during last two decades. One of the most known chaotic circuits is the Chua's circuit $[7,8]$ containing four linear elements (two capacitors, one resistor and one inductor) and a nonlinear resistor called Chua's diode constructed using operational amplifiers. An integrated implementation of Chua's circuit was presented in [9] using a $2 \mu \mathrm{m}$ CMOS technology, and occupying a silicon area of $2.5 \times$ $2.8 \mathrm{~mm}^{2}$. Other researchers $[10,11,12]$ present different chaotic oscillators using inductances, resistors and nonlinear elements (similarly to Chua's circuit) requiring the use of several operational amplifiers, with a considerable impact on the circuit area. 
To overcome those area problems a simpler design has been recently introduced using a total of 20 MOS transistors [13]. The circuit implements the double-scroll chaotic equation and its effectiveness has been demonstrated using SPICE simulations. More recently a transistor-based chaotic circuit has been proposed [14]. The circuit uses a bipolar transistor, two capacitors and two resistors, but requires the use of an external sinusoidal oscillator for its proper operation.

In this work we present an autonomous low-cost chaotic Integrated Circuit (IC). A low area of integration is achieved due to the use of a relatively low number of transistors. The chaotic behavior is reproduced implementing a N-shaped iteration map using three coupled neurons designed with a simple CMOS scheme (see [15] for a detailed description of how the N-shaped iteration map may reproduce a chaotic behavior). The circuit was implemented on a $0.35 \mu \mathrm{m}$ technology and integrated in $47 \mu \mathrm{m} \times 57 \mu \mathrm{m}$ of silicon area. It generates a digital output that can be used to create a true random bit sequence. The proposed circuit is, to the best of our knowledge, the smallest chaotic integrated circuit that has been built. The rest of this paper is organized as follows: in section 2 we show the basic principles of the proposed chaotic system, in section 3 we show the experimental results and finally, in section 4 we present the conclusions.

\section{Chaotic neural network design}

The proposed design obtains the chaotic signal from a small neural network. Neurons are non-linear elements that implement a sigmoid-like function $(f(x))$ to the result of adding and weighting all the neuron inputs $I_{i}$.

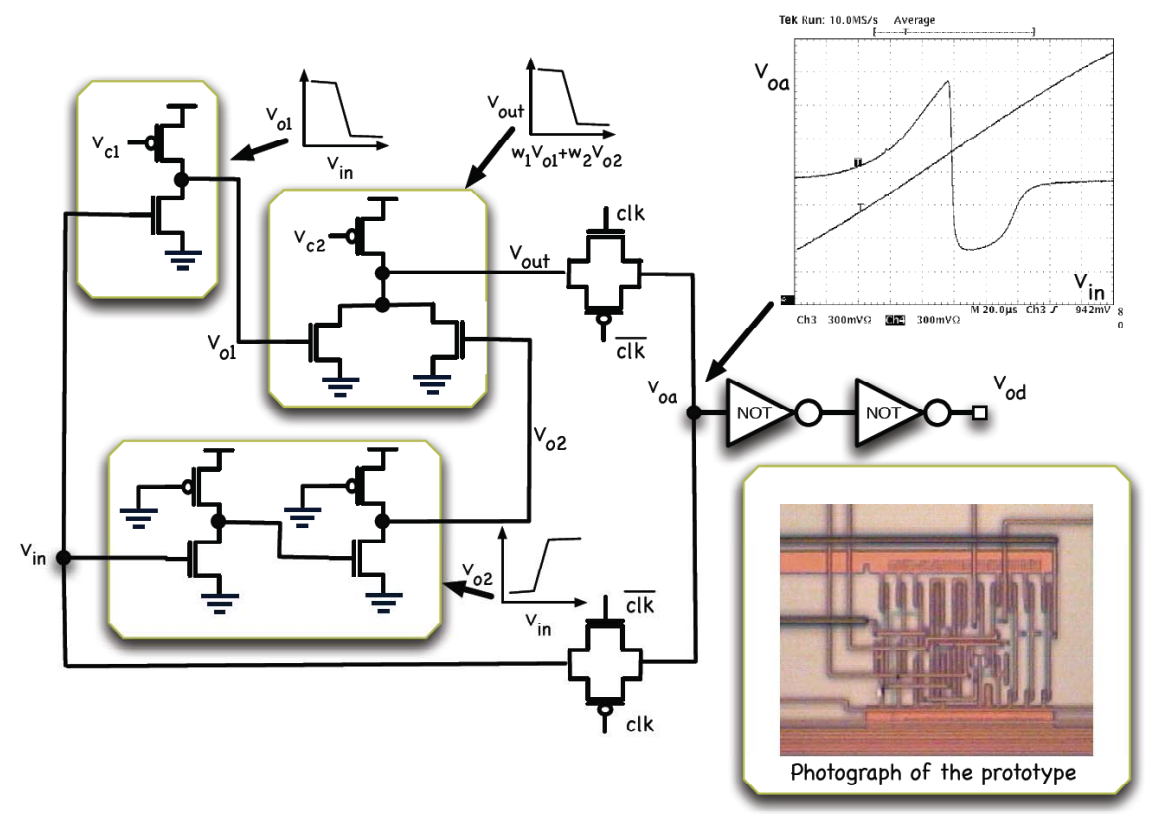

Fig. 1. Circuit implementation of the chaotic neural network. Two outputs, an analog and a digital signal $\left(V_{o a}\right.$ and $\left.V_{o d}\right)$ are generated. 

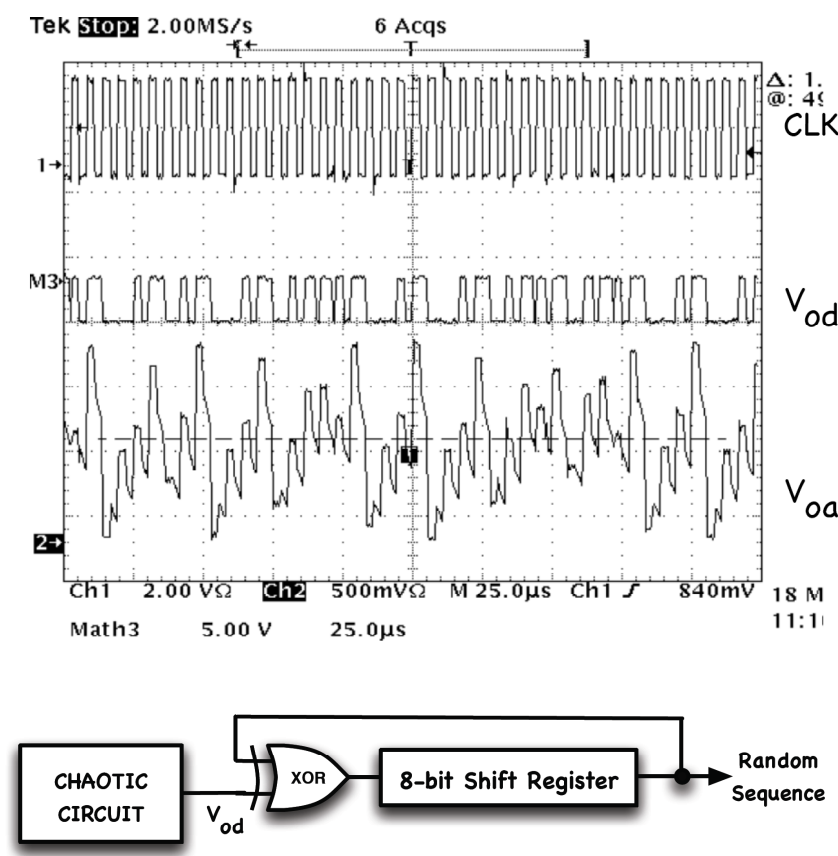

(b)

Fig. 2. (a) Dynamic characteristics for the circuit when $V_{c 1}=0 \mathrm{~V}$ and $V_{c 2}=0.68 \mathrm{~V}$. An analog (bottom signal) and a digital output (middle signal) are obtained. The threshold level of inverters connected to $V_{o a}$ is shown as a dashed line with $V_{o a}$. (b) Circuit scheme for random bit generation.

The neuron response $(y)$ is therefore expressed as follows:

$$
y=f\left(\sum_{i} \omega_{i} I_{i}\right)
$$

To reproduce a chaotic behavior, we designed a small network with three neurons to implement a $\mathrm{N}$-shaped transfer function $N(x)$ as it has been reported that, under specific conditions, iterations on a N-shaped map may reproduce a chaotic behavior (see [15]). The state of the system $x_{n}$ is updated at each iteration through the N-shaped transfer function $N\left(x_{n}\right)$ so that:

$$
x_{n+1}=N\left(x_{n}\right)
$$

The function $N(x)$ may be reproduced with three coupled neurons, each one implemented using MOS transistors (see Fig. 1). Each neuron circuit is reproducing Eq. (1) with pre-defined values for the input weights $\left(\omega_{i}\right)$ that depend on transistor sizing (see Fig. 1 to see the specific transfer function of each neuron). We also included two controlling voltages $\left(V_{c 1}\right.$ and $\left.V_{c 2}\right)$ to change the PMOS resistance of two neurons of the circuit, thus modifying the transfer function of the whole system. A transfer function similar to a sigmoid is obtained at the output of each neuron due to the non-linear characteristics of MOS devices. The channel width of NMOS transistors has been selected to obtain the proper weight for the inputs. The transfer curve obtained by the whole neural network depends on the selected values for $V_{c 1}$ 
and $V_{c 2}$. For specific values of these controlling voltages we can obtain an Nshaped transfer function that allows the reproduction of the chaotic behavior. A measurement of the N-shaped transfer function of the fabricated circuit is shown in Fig. $1\left(V_{o a}\left(V_{i n}\right)\right)$.

The circuit is designed to provide two signals, one analog and the other digital $\left(V_{o a}\right.$ and $\left.V_{o d}\right)$. Two transmission gates controlled by an external clock are serially connected to the neural network. When the clock signal is low, the voltage $V_{o a}$ is evaluated by the network while when the clock is high the iteration $N\left(V_{o a}\right)$ replaces the previous $V_{o a}$ value. The final result is the iteration of Eq. (2) at each clock cycle. Finally $V_{o d}$ is generated from $V_{o a}$ using CMOS inverters.

The proposed circuit was fabricated using a $0.35 \mu \mathrm{m}$ standard CMOS process. The nominal supply voltage is $3.3 \mathrm{~V}$ while the total circuit dimensions are $47 \mu \mathrm{m} \times 57 \mu \mathrm{m}$. A photograph of the fabricated prototype is included as an inset in Fig. 1.

\section{Experimental results}

In Fig. 1 we show the measured transfer function of the system when the controlling voltages are settled to specific voltage values. We also provide the identity function $(y(x)=x)$ since the output signal is chaotic only when the intersection between such a function and $N(x)$ occurs when $N(x)$ is decreasing [15]. Parameters $V_{c 1}$ and $V_{c 2}$ were fixed to $0 V$ and $0.74 V$ respectively.

In Fig. 2 (a) we show the dynamic behavior of the circuit when the clock signal oscillates at $0.18 \mathrm{MHz}$. Two non-periodic signals $\left(V_{o d}\right.$ and $\left.V_{o a}\right)$ are obtained. From the digital signal $\left(V_{o d}\right)$ we can generate a true random signal. In Fig. 2 (b) we show a circuit configuration that can be used to obtain a true random sequence that pass the FIPS-140-2 test suite (standardized test to accredit the quality of random bit sequences). A 8-bit shift register is serially filled with the product of performing the XOR function of $V_{o d}$ and the output bit of the register. In contrast to pseudo-random signal generators as LFSR counters, the bit sequence generated does not present a periodic behavior (an LFSR of $n$-bits presents a period of repetition of $\left.2^{\mathrm{n}}-1 \mathrm{bits}\right)$. True random bit streams are ideal to implement stochastic-based computations $[16,17]$ in which true random sequences are needed to generate high quality stochastic signals [17].

In Fig. 3 (a) we show that the fabricated circuit present the phenomena of period doubling bifurcation cascade. The bifurcation diagram is obtained varying the controlling voltage $V_{c 1}$ between 0 and 1.6V. Fig. 3 (a) has been obtained by plotting the $V_{o a}$ signal vs. $V_{c 1}$ at each iteration of Eq. (2) $\left(V_{o a}\left(V_{c 1}\right)\right)$. Each division of the oscilloscope is equivalent to $200 \mathrm{mV}$. We computed the Lyapunov exponents of the dynamic system when varying the controlling voltage $V_{c 2}$. Lyapunov exponents constitute a useful metric for the estimation of the system sensitivity to initial conditions. The level of chaos is directly related to the Lyapunov exponent values since positive (negative) exponents are related to chaotic (periodic) behavior. Two trajectories with 


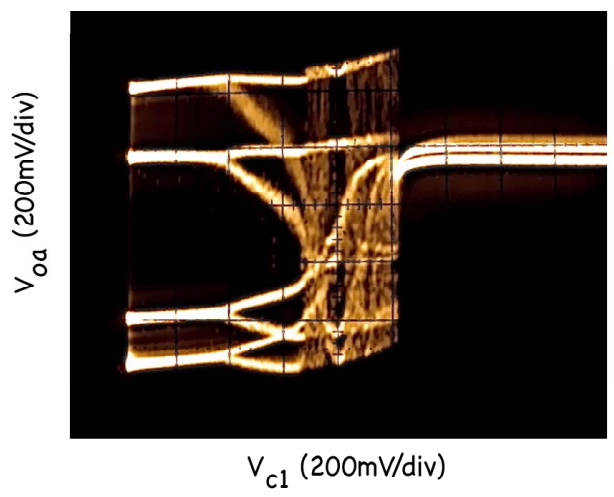

(a)

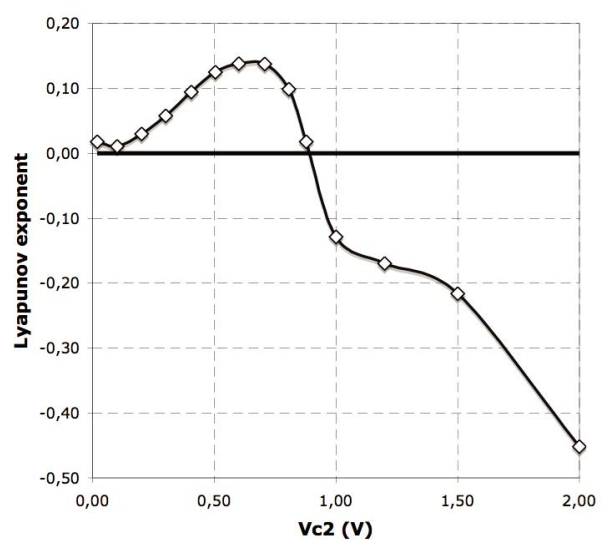

(b)

Fig. 3. (a) Bifurcation diagram obtained when varying $V_{c 1}$ (plot of $V_{o a}$ vs. $\left.V_{c 1}\right)$ (b) Lyapunov exponent value as a function of the controlling voltage $V_{c 2}$.

nearby initial conditions diverge, on average, at an exponential rate characterized by the Lyapunov exponent. Therefore, the separation between two trajectories $(\delta x)$ presents a time evolution given by:

$$
\left|\delta x_{k}\right|=\left|\delta x_{0}\right| e^{\lambda k}
$$

where $\delta x_{k}$ is the separation between the two trajectories in the k-th iteration, and $\delta x_{0}$ is the initial separation. We computed the Lyapunov exponents using the technique described in [18] for different values of $V_{c 2}$ (see Fig. $3(\mathrm{~b})$ ) when $V_{c 1}$ is fixed to $0 V$. It is shown that $\lambda$ is positive (and therefore the behavior is chaotic) when $V_{c 2}<0.9 \mathrm{~V}$.

\section{Conclusion}

A simple fully-CMOS neural network circuit exhibiting a chaotic behavior has been presented and validated experimentally on a $0.35 \mu \mathrm{m}$ technology. The circuit presents a small area of integration and is implemented using MOS transistors only. The chaotic nature of the circuit is demonstrated experimentally through the computation of the Lyapunov exponents and the bifurcation diagram. To the best of our knowledge this is the smallest chaotic integrated circuit that has been built. The circuit is able to reproduce a 
true random bit sequence that can be used to implement stochastic-based computations [17].

\section{Acknowledgments}

This work was supported by the Balearic Islands Government in part by the Regional European Development Funds (FEDER) under project PROGECIB$32 \mathrm{~A}$. 